\title{
ASSESSMENT OF FACTORS INFLUENCING ADEQUACY OF NATIVE ARTERIOVENOUS FISTULA FOR LONG TERM VENOUS ACCESS IN HEMODIALYSIS PATIENTS.
}

Shahid Abbas, Sharad Khandelwal, Riaz Hussain, Zubin Gandhi, Himanshu Dagor, Pranay Bajpai, Gunjan Shrivatava, R. K. Jha.

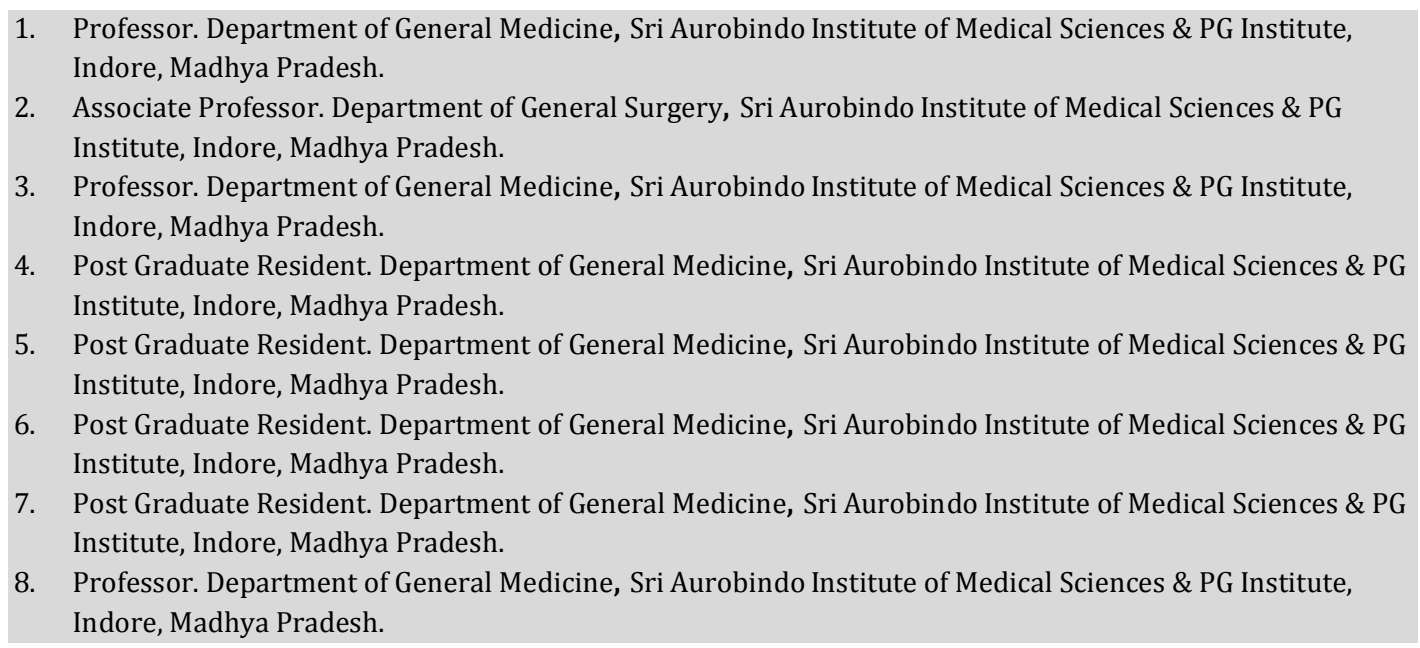

\section{CORRESPONDING AUTHOR:}

Dr. Shahid Abbas,

21, Babji Nagar,

Behind Sagar automobiles near Shalimar township,

A. B. Road, Near Dewas Naka. Indore (M.P.).

E-mail: drshahidabbas786@gmail.com

ABSTRACT: OBJECTIVE:-The aim of our study was to assess the factors affecting adequacy of arteriovenous fistula (AVF) and to correlate it with clinical and demographic factors. MATERIAL AND METHODS: The prospectively designed study was conducted to determine adequacy of AVF in 130 patients over a period of two years. Adequacy of AVF was graded if the blood flow rate was higher or equal to $300 \mathrm{ml} / \mathrm{min}$ on at least fifty percent of dialysis sessions in one month. AV-fistula adequacy was correlated with clinical and demographic factors like age, gender, diabetic status, smoking status, body mass index (BMI), serum calcium, serum phosphorus and Ca $\times \mathrm{P}$ product, serum albumin and an evaluation for peripheral vascular disease (PVD). RESULTS: The study included 130 patients (68 males and 42 females). Early adequacy rate of 110 fistulas was $66 \%$ and $51 \%$ at end of $3^{\text {rd }}$ and $6^{\text {th }}$ months respectively. Diabetes was present in $41 \%, 32 \%$ were hypertensive and $27 \%$ of the patients were overweight (BMI $\geq 27$ $\mathrm{kg} / \mathrm{m}^{2}$ ). The adequacy rate was lower in older (age $\geq 65$ ) patients ( $34.0 \mathrm{vs.} 57.6 \%, \mathrm{P}=0.028$ ), in overweight (BMI $\geq 27 \mathrm{~kg} / \mathrm{m}^{2}$ ) patients (33.3 vs. 57.5\%, P =0.026). It was also marginally lower in diabetics versus nondiabetics ( 40.0 vs. $58 \%, \mathrm{P}=0.058)$ and the presence of peripheral vascular disease and increase calcium phosphorus product adversely affected AVF adequacy. The adequacy rate was not affected by patient smoking status and serum albumin. The adequacy rate was substantially lower for forearm versus upper arm fistulas $(40.0$ vs. $64 \%, \mathrm{P}=$ $0.0131)$. The adequacy of forearm fistulas was particularly poor in women (13\%), patients age 65 or older $(12.5 \%)$, and diabetics $(24 \%)$. In contrast, upper arm fistulas were adequate in 
$59.2 \%$ of women, $56.3 \%$ of older patients, $54 \%$ of diabetics, and $30 \%$ of those having moderate to severe PVD. CONCLUSIONS: Fistula adequacy and survival appear to be dependent on individual comorbid conditions and the integrity of the vasculature. Discriminant AVF site selection and adequate preoperative assessment of the vasculature remain crucial to AVF survival.

KEY WORDS: - arteriovenous fistula, adequacy, upper arm, forearm, diabetes, overweight, gender, peripheral vascular disease, chronic renal failure.

INTRODUCTION: Arteriovenous fistula is the treatment of choice for haemodialysis in chronic renal failure patients. Back in early 20th century the arteriovenous shunting was started for haemodialysis.(1) In 1966 Brescia, Cimino, and Hurlwith surgically created fistulae between cephalic vein and radial artery at the wrist.(2-3) Currently it is the mainstay of treatment of chronic kidney disease patients. These arteriovenous fistula have enormous benefits in term of their safety profile and long term patency.(4-5) Chronic renal failure patients may have unsuitable veins for native arteriofistula. ${ }^{(6)}$

Recent guidelines published by the National Kidney Foundation Dialysis Outcome Quality Initiative (DOQI) on vascular access has suggested that for patients requiring chronic haemodialysis, the preferred site for access is a native arteriovenous fistula (AVF).(7) The recommendation of DOQI may cause an aggressive approach for creation of fistula rather than a graft. Although fistulae have longer patency as compared to grafts, a substantial number of fistulae do not function adequately either due to failing maturation or to thrombosis directly after the operation. Severe arteriosclerosis, edema of the arm, vascular calcification, multiple vein puncture do pose problems. ${ }^{(8)}$ To overcome these difficulties other fistula sites in different parts of body have been recommended. Selection of brachial artery poses risk of significant cardiovascular complications moreover this site provides limited space for venipuncture for haemodialysis purpose.(9-10) Patients with end-stage-renal-disease (ESRD) usually have a number of co-morbid conditions in addition to their renal disease, so they should be referred early for arteriovenous fistula (AVF) formation. (11)

MATERIAL AND METHODS: This prospectively designed case series study was conducted at Department of Medicine (Nephrology Division), Sri Aurobindo Institute of Medical Sciences, Indore (M.P.) from 1st January 2010 to 31st December 2011. Data sheets were generated for accurate documentation of dates of AVF creation, anatomic locations, dates of failure or cannulation, patient demographics, and records of salvage attempts on failing fistula. Before the start of the study by the end of year 2009 we had 180 chronic kidney disease (CKD) stage 5 patients on haemodialysis. In the year 2010 and 2011 we had 121 and 106 new patients respectively, of which ninety nine CKD stage 5 patients in 2010 and ninety CKD stage 5 patients in 2011 who were not on haemodialysis were put on haemodialysis and advised AVF surgery. Rest of the patients were already on haemodialysis, came or were referred to our institute from other centres and hospitals. Of the total patients (i.e.301) in 2010, fourty patients stopped dialysis and didn't reported and thirty five patients on haemodialysis died and four patients opted for ambulatory peritoneal dialysis. Out of the 328 patients in 2011, thirty nine patients on haemodialysis died, 45 patients stopped dialysis and 15 opted for ambulatory peritoneal dialysis. Thus effectively 222 and 229 CKD stage 5 patients were on chronic dialysis at the end of year 2010 and 2011 respectively. The demographics of our total CKD stage 5 dialysis patient population during the above study period are as follows: $32 \%$ of the patients are age 65 or 
older. Forty-nine percent of the patients are female. Fourty one percent of the patients have diabetes, $35 \%$ had hypertension and $18 \%$ had both hypertension and diabetes.

One of our specific goals has been a concerted effort to increase the construction of an $A-V$ fistula in preference to a graft, whenever suitable vessels could be found. If after physical evaluation, it was believed that the forearm vessels were inadequate for the creation of a radio cephalic fistula or if a previous forearm fistula failed to mature, an attempt to construct an upper arm fistula was done. A graft was constructed only if the patient had no suitable vessels for a fistula or if an upper arm fistula had failed to develop.

After the evaluation of normal radial pulse and distensible veins at the wrist of nondominant arm, arteriovenous fistula at wrist was performed, after explaining the patient about the AVF procedure and taking written consent. Patients undergoing other types of vascular access or secondary fistula formation were excluded. Pre-operative evaluation included detailed evaluation of vessels clinically, quality of artery and vein, pin pointing the main arterial supply of the hand by Allen's test. Status of the arm whether dominant or non-dominant (some patients could be having left dominant arm), functional or paralysed arm, previous vein puncture or edema or trauma were assessed. All the fistula formation were performed under local anaesthesia. Transverse incision was made in antecubital fossa to expose Brachial artery, Basilic and Cephalic vein and vertical incision was made at forearm for Radial artery and Cephalic vein. Anastomosis was created in an end to side or side-to-side fashion depending upon mobility of target vein.

A minimum of one month after fistula creation was required prior to the first venipuncture. New fistulas were initially used for single needle dialysis. If this was successful, they were used with two needles at low blood flow $(200 \mathrm{ml} / \mathrm{min})$, with the blood flow increasing gradually over a period of several weeks.

We defined fistula adequacy by modification of the protocol used by Miller et al.(12) This was defined prospectively as the ability to use it for haemodialysis with two needles and a blood flow of at least $300 \mathrm{ml} / \mathrm{min}$ on at least fifty percent of dialysis sessions in one month. A fistula was considered inadequate if it (a) clotted before it could be used, (b) was still not usable for dialysis six months after its construction, or (c) was converted electively to an A-V graft prior to being used for dialysis. Fistula adequacy was deemed indeterminate if the patient died, received a kidney transplant, or was lost to follow-up before the fistula could mature, or if the fistula had not yet matured at the time of analysis but had less than six months of follow-up.

The following demographic and clinical information was collected for each patient: age, sex, diabetic status, smoking status, body mass index (BMI), serum calcium, serum phosphorus, serum albumin. BMI was calculated from the patient height and weight using standard formulae. Patients with a BMI $\geq 27 \mathrm{~kg} / \mathrm{m}$ were considered overweight. A patient was considered a smoker if he or she had ever smoked. Peripheral vascular disease (PVD) was assessed by the Edinburgh Claudication Questionnaire as simple screening method in combination with ankle brachial index.(13)

STATISTICAL ANALYSIS: Chi-square test for associations was used to test for the association between adequacy of fistula and demographic characteristics of the patients and for the association between adequacy of fistula and site of placement (upper arm vs. forearm). Because of the significance of site of fistula placement, associations between site of placement and fistula adequacy were examined within specific subgroup of the patients. The statistical significance was set at $\mathrm{p}<0.05$. 
RESULTS: During the two-year study, 130 patients underwent A-V fistula formation. Ten patients died and ten were lost to follow-up so the adequacy of the remaining 110 fistulas could be determined and was the subject of further statistical analysis.

Medical history for causes of chronic kidney disease who underwent AV fistula surgery included diabetes in 45 (41\%), hypertension in 32 (29\%) patients and 16 (14.5\%) had both history of hypertension and diabetes mellitus. Other associated diseases were found in 17 (15\%). These were obstructive nephropathy 05 (4.5\%), chronic glomerulonephritis 05 (4.5\%), chronic interstitial nephritis $05(4.5 \%$,) renovascular disease $01(0.9 \%)$ and polycystic disease 01 (0.9\%).(Table 1 )

Of the 110 fistulas for which adequacy could be determined, 56 (51\%) developed adequately, as defined by our prospective criteria, to be used for dialysis with a blood flow of at least $300 \mathrm{ml} / \mathrm{min}$. Of those that did not matured adequately, 22 fistulas developed early thrombosis and 32 fistulas did not mature within six months of their creation or were electively converted to grafts. The mean time from fistula placement to adequacy for dialysis was $2.1 \pm 1.2$ months. Among the fistulas that achieved adequacy for dialysis, the patency rate was 96,87 , and $80 \%$ at 6,9 , and 12 months, respectively.

The relevant demographic and clinical characteristics of the patients for whom fistula adequacy could be determined are summarized in Table 2 . The patients' age (mean \pm SD) was $50 \pm 12$ years and ranged between 18 and 74 years. Seventy one percent of the fistulas were created in patients 65 years of age or younger. Approximately $62 \%$ of the fistulas were constructed in men and 38\% in women. Diabetes was present in $41 \%$ of the patients, and $44 \%$ were smokers. Approximately $27 \%$ of the patients were overweight (BMI $\geq 27 \mathrm{~kg} / \mathrm{m}^{2}$ ).

The mean serum albumin concentration was $3.2 \pm 0.8 \mathrm{~g} / \mathrm{dl}$ and ranged between 1.6 and $4.4 \mathrm{~g} / \mathrm{dl}$. The serum albumin was $<3.5 \mathrm{~g} / \mathrm{dl}$ in $55 \%$ of the patients.

The likelihood of fistula adequacy differed substantially among various demographic and clinical subgroups (Table 2). The fistula adequacy rate was lower in forearm fistulas as compared to upper arm fistulas ( $40 \%$ vs. $64 \%, p=0.0131$ ), in older patients (age $\geq 65,34.3 \%$ vs. $57.6 \%$, Odds ratio $0.38, \mathrm{p}=0.028$ ),in overweight patients (BMI $\geq 27 \mathrm{~kg} / \mathrm{m}, 33.3 \%$ vs. $57.5 \%$, Odds ratio $0.37, \mathrm{p}=0.026)$ and in diabetics ( $40 \%$ vs. $58.4 \%$, Odds ratio $0.47, \mathrm{p}=0.058)$. The presence of PVD was significantly associated with the reduced fistula adequacy (25\% vs. 56,4\%, Odds ratio $0.27, \mathrm{p}=0.0324$ ). An increase in Cax $\mathrm{P}$ product $>72$ also adversely affected AVF adequacy ( $41 \%$ vs. $59.3 \%, p=0.06$, Odds ratio 0.48 ). The observed fistula adequacy rate in overweight patients was lower in both diabetic and nondiabetic patients. The fistula adequacy rate was not affected by smoking status and the serum albumin levels.

Sixty fistulas were placed in the forearm, and 50 were placed in the upper arm. The site of the fistula was an important predictor of adequacy (Table 3). The fistula adequacy rate was nearly twice as high for upper arm fistulas as compared with forearm fistulas. The discrepancy between upper arm and forearm fistulas was particularly striking for certain subgroups of patients. In comparison to forearm fistula the upper arm fistulae adequacy rates was substantially higher in following subgroup $54.2 \%$ vs. $24 \%$ in diabetics, $59.2 \%$ vs. $13.3 \%$ in females, $56.3 \%$ vs. $12.5 \%$ in patients aged $\geq 65$ years and $53.3 \%$ vs. $24 \%$ in those having a an increase in Cax P product $\geq 72$. Moderate to severe peripheral vascular disease (PVD) had a negative effect on AVF construction and quality with an adequacy rate of only $25 \%$ with only upper arm fistula's adequacy rate of $40 \%$. Nondiabetic men under the age of 65 were the only subset of haemodialysis patients in whom there was a substantial likelihood (50\%) of adequacy for a forearm fistula. 
In our subgroup analysis of 110 fistulas (Table 4), the maturation and adequacy rate at end of 3 months showed $72(66 \%)$ adequate fistulas with a failure rate of $34 \%$ which increased to $49 \%$ as defined by our prospective criteria of adequately working fistula. Therefore, of total failed fistulas, $70 \%$ failure rate was in the first three months and main cause of failure was older age, PVD, obese, diabetics and females.

Among the fistulas analyzed in this study, 51\% represented a first access in a dialysis patient (primary fistula), and 49\% did not develop adequately were a subsequent access placed after the failure of a previous fistula or graft (secondary fistula).

DISCUSSION: The major complication with AVF is the high frequency of primary failure or suitability failure due to early thrombosis or lack of maturation.(14-16) Primary AVF failure is reported to occur after AVF placement in up to $40 \%$ cases. (14-16) There are many factors associated with the high rate of primary AVF failure. The dialysis population is growing exponentially with older patients and a higher proportion of diabetes and other comorbidities, all of these factors may increase the likelihood of vascular disease and decrease the likelihood of fistula maturation. Multiple studies have confirmed that older age, coronary artery disease (CAD) and peripheral vascular disease (PVD) are clinical indicators associated with an increased risk for AVF failure.(17-19) This makes pathophysiologic sense because adequate inflow and outflow are required for AVF maturation and would be hampered by diseased vasculature represented by atherosclerosis and arteriosclerosis. ${ }^{(18)}$ We observed a primary patency and adequacy rates of $66 \%$ and $51 \%$ at three and six months respectively in our patients. The overall incidence of early failure of AVF's in our study was $49 \%$, a bit on higher side as we followed a stringent criterion for AVF adequacy. The range of early failure of AVF's in other published series is from $24 \%$ to $51 \%$. The early failure rate in our study is considerably higher than the 24 to $27 \%$ rate reported in two previous studies $(20,21)$ as they had fewer comorbid conditions. all of these factors may increase the likelihood of vascular disease and decrease the likelihood of fistula maturation. Early clotting of fistulas was reported to occur in 9 to $12 \%$ of cases in three separate reports ${ }^{(22-24)}$, a rate lower than the $20 \%$ thrombosis rate $(22$ of 110 fistulas) observed in this study. Early fistula adequacy was more rigorously defined in our study to sustain a blood flow of at least a consistent $300 \mathrm{ml} / \mathrm{min}$. It is possible that some marginal fistulas will be able to deliver a lower blood flow during dialysis. Thus, some fistulas that were deemed adequate in studies previously might not have met the definition of adequacy used in our current study.

In an older study, Wong $\mathrm{V}$, et al; described that there was poor outcome of fistulas in women, which may be partly explained by their smaller vessels.(25) In our study we found out that in females, AVF were more successful and adequate $(59.2 \%)$ when constructed in upper arm. The recent evidence from national and international studies deny any affect of sex on duration of patency of arteriovenous fistulae.(26,27) Patients with post-operative fistula failure due to infection or hematoma were not destined to salvage procedure as international studies reveal disappointing results, therefore fistula ligation was preferred in these patients followed by reestablishment of new arteriovenous fistula at suitable other site.(28,29)

In our study also diabetes was a significant risk factor for occurrence of early failure of AVF. Leapmen and colleagues (30) also demonstrated the poor out come for diabetic patients and reported patency rates of $42 \%$ at 12 months and $18 \%$ at 60 months. Similarly, Monroy-Cuadros $\mathrm{M}$ et al (31) found diabetes to be a significant risk factor for failure of fistulas within the 1st year. Diabetic patients also have a poor out comes with prosthetic fistula. The poorer results in 
diabetics might be explained by high prevalence of peripheral arterial disease in this population. Calcification of the blood vessels enhanced by an increase in calcium phosphorus product also seems to play a role in early failure of AVF due to reduced arterial compliance and impaired arterial dilation necessary for the maturation of a fistula. Furthermore, diabetes is associated with endothelial dysfunction (32) and overall increase in oxidative stress that may explain the increased incidence of thrombotic events. It could also be due to infectious complication because of immunosuppression in addition sclerosed arteries also make anastomotic techniques difficult.

A distal AVF carries a higher rate of early failure in several studies. This is mainly due to vessels of small calibre and damage to the vessel wall due to venipunctures. The etiology of AVF primary failure is not limited to pathophysiologic processes. Public healthcare policy, practitioner training, available interventions and patient education all contribute to the success of a functioning fistula. Worldwide initiatives have demonstrated the potential beneficial impact of public policy on improving outcomes and decreasing costs.(33)

The most effective way to reduce the need for repeated dialysis access procedures is to place native A-V fistulas as often and as soon as possible. The Fistula First Initiative (11) is dedicated to improving care for people with chronic kidney disease by timely referral to a nephrologist and to a surgeon increasing AVF placement and thus avoiding central catheter induced complications. ${ }^{(33)}$ Literature review indicates that with early referral to a surgeon and consequent preoperative vessel mapping, some type of fistula can be placed in up to $75 \%$ of patients. $(34,35)$

At our institution, we were able to substantially increase the proportion of fistulas placed in new haemodialysis patients. That we were aggressive in attempting fistula placement is reflected in the fact that the proportion of fistulas placed in diabetics (41\%) was similar to the proportion of diabetics in our dialysis population (41\%). Similarly, the proportion of fistulas placed in older (age $>65$ ) patients $(30 \%)$ was similar to the proportion of older patients in our dialysis population (32\%). Interestingly, the proportion of fistulas placed in female patients (38\%) was considerably lower than the proportion of women in our overall dialysis population (49\%), which is consistent with previous observations. (34) Clearly, a more aggressive approach to the creation of $\mathrm{A}-\mathrm{V}$ fistulas may result in an increased number of failures in patients with marginal vascular anatomy.

The DOQI guidelines recommend creating a forearm fistula in preference to an upper arm fistula. (7) This study suggests that for many haemodialysis patients (women, diabetics, or age $\geq 65$ years and those with peripheral vascular disease), the likelihood of patency of a forearm fistula is so low (Table 3 ) that they will very likely require a second dialysis access procedure. Moreover, if the patient has already initiated dialysis, this would require a prolonged period of dialysis with a temporary catheter and its associated problems of frequent infection, thrombosis, and inadequate dialysis. Thus, in these subsets of patients, it might be reasonable to construct the initial fistula in the upper arm rather than the forearm. Such a strategy would maximize the probability that the first fistula constructed would be usable for dialysis. This would not only improve outcome but also decrease cost. For example, in this study, 13 of 15 forearm fistulas constructed in female patients were inadequate (Table 3). If the proposed strategy was followed for the patients analyzed in this study, these 13 women would have had an upper arm fistula constructed as their initial dialysis access. If the upper arm fistula was inadequate, it could then be converted to an A-V graft. An alternative strategy would be to construct preferentially forearm A-V grafts or an upper arm A-V fistula. We believe that only in 
selected subpopulations (nondiabetic men under age 65) is the adequacy rate of a forearm fistula sufficiently high (56\%) to justify attempting the first fistula at that site.

The recent description of preoperative sonographic venous mapping offers promise for increasing fistula adequacy. (36) Even when fistulas are constructed in the upper arm, over one third of them will be inadequate (Table 3), necessitating the creation of another dialysis access. This observation highlights the need to refer predialysis patients for fistulas sufficiently early (approx 6 months prior to the anticipated date for initiation of dialysis) to permit a determination of whether the fistula will develop adequately, and the opportunity to consider a second access if the fistula fails to mature.

CONCLUSION: As the dialysis population is growing exponentially with older patients and a higher proportion of diabetes, hypertension and other comorbidities, all of these factors may increase the likelihood of vascular disease and decrease the likelihood of fistula maturation. The discrepancy between upper arm and forearm fistulas was particularly striking for certain subgroups of patients. Therefore, to achieve an optimal outcome with A-V fistulas, we recommend that they be constructed preferentially in the upper arm in female, diabetic, and older haemodialysis patients and in those having moderate to severe PVD. Nondiabetic men $<65$ years of age were the only subset of haemodialysis patients in whom there was a substantial likelihood of adequacy for a forearm fistula. Key to success is early referral and early establishment of a vascular access after assessment. Regular monitoring of the fistula is indicated, and fistula flow should be assessed in critical cases as the single most important predictor of fistula thrombosis. It must be the aim to prevent thrombosis rather than to intervene on established thrombosis.

ACKNOWLEDGMENT: We are thankful to Dr.Vinod Bhandari, Chairman, Sri Aurobindo Medical College and Postgraduate Institute, Dean Dr.S.D.Joshi and Dr. R.K. Jha, Professor and Head of Department of General Medicine for the permission to publish this original article.

\section{REFERENCES:}

1. Quinton W, Dillard D, Scribner BH. Cannulation of blood vessels for prolonged haemodialysis. Trans Am Soc Artif Intern Organs. 1960; 6:104-113.

2. Brescia MJ, Cimino JE, Appel K, Hurwich BJ. Chronic haemodialysis using venipuncture and a surgically created arteriovenous fistula. N Engl J Med. 1966; 275(20):1089-1092.

3. Pflederer TA, Kwok S, Ketel BL, Pilgram T. A comparison of transposed brachiobasilic fistulae with non-transposed fistulae and grafts in the Fistula First era. Semin Dial. 2008; 21(4):357-363.

4. Goodkin DA, Pisoni RL, Locatelli F, Port FK, Saran R. Haemodialysis vascular access training and practices are key to improved access outcomes. Am J Kidney Dis. 2010; 56(6):1032-42.

5. Ahmed GM, Mansour MO, Elfatih M, Khalid KE, Mohammed Ahmed ME. Outcomes of arteriovenous fistula for haemodialysis in Sudanese patients: Single-center experience. Saudi J Kidney Dis Transpl. 2012; 23(1):152-157.

6. Saran R, Elder SJ, Goodkin DA, Akiba T, Ethier J, Rayner HC, et al. Enhanced training in vascular access creation predicts arteriovenous fistula placement and patency in hemodialysis patients: Results from the Dialysis Outcomes and Practice Patterns Study. Ann Surg. 2008; 247(5):885-891. 
7. NKF-DOQI Clinical Practice Guidelines for Vascular Access. Am J Kidney Dis. 2006 Jul;48 Suppl 1:S248-73.

8. Keith DS, Nichols GA, Gullion CM. Longitudinal follow up and outcomes among a population with chronic kidney disease in a large managed care organization. Arch Int Med. 2004; 164(6):659-663.

9. Kim YO, Yang CW, Yoon SA. Access blood flow as a predictor of early failure of native arteriovenous fistula in haemodialysis patients. Am J Nephrol. 2001; 21(3):221-225.

10. Kirksey L. Unrecognized high brachial artery bifurcation is associated with higher rate of dialysis access failure. Semin Dial. 2011; 24(6):698-702.

11. Vachharajani TJ, Moossavi S, Jordan JR, Vachharajani V, Freedman BI, Burkart JM. Reevaluating the Fistula First Initiative in Octogenarians on Haemodialysis. Clin J Am Soc Nephrol. 2011; 6:1663-1667.

12. Miller PE, Tolwani A, Luscy CP, Deierhoi MH, Bailey R, Redden DT. Predictors of adequacy of arteriovenous fistulas in haemodialysis patients. Kidney Int. 1999 Jul;56(1):275-80.

13. Chen HW, Liu HE. Peripheral arterial occlusive disease and its care in haemodialysis patients. Hu Li Za Zhi. 2011 Apr; 58(2):16-21.

14. Ravani P, Spergel LM, Asif A, Roy-Chaudhury P, Besarab A. Clinical Epidemiology of arteriovenous fistula in 2007. J Nephrol.2007; 20:141-149.

15. Maya ID and Allon M. Vascular access:Core curriculum 2008.Am J Kid Dis. 2008; 51:702708.

16. Maya ID, O’Neal JC, Young CJ, Barker-Finkel J, Allon M. Outcomes of brachiocephalic fistulas, transposed brachiobasilic fistulas, and upper arm grafts. Clin J Am Soc Nephrol 2009;4:86-92.

17. Allon M. Current management of vascular access. Clin J Am Soc Nephrol .2007; 2:786800.

18. Lok CE. Fistula First intiative: Advantages and pitfalls. Clin J Am Soc Nephrol.2007; 2:1043-1053.

19. Lok CE, Allon M, Moist LM, Oliver MJ, Shah H, Zimmerman D. Risk equation determining unsuccessful cannulation events and failure to maturation in arteriovenous fistula. J Am Soc Nephrol. 2006 ;17: 3204-3212.

20. Winsett OE, Wolma FJ: Complications of vascular access for haemodialysis. South Med J.1985; 78:513-517.

21. Palder SB, Kirkman RL, Whittemore AD, Hakim RM, Lazarus JM, Tilney NL: Vascular access for haemodialysis: Patency rates and results of revisions. Ann Surg.1985; 202:235-239.

22. Bender MHM, Bruyninckx MA, Gerlag PGG: The brachiocephalic elbow fistula: A useful alternative angioaccess for permanent haemodialysis. J Vasc Surg. 1994; 20:808-813.

23. Kherlakian GM, Roedersheimer LR, Arbaugh JJ, Newmark KJ, King LR: Comparison of autogenous fistula versus expanded polytetrafluoroethylene graft fistula for angioaccess in haemodialysis. Am J Surg.1986; 152:238-243.

24. Bonalumi U, Civalleri D, Rovida S, Adami GF, Gianetta E,Griffanti-Bartoli F: Nine years' experience with end-to-end arteriovenous fistula at the "anatomic snuffbox" for maintenance haemodialysis. Br J Surg.1982; 69:486-488. 
25. Wong V, Ward R, Taylor J, Selvakumar S, How TV, Bakran A. Factors associated with early failure of arteriovenous fistulae for hemodialysis access. Eur J Vasc Endovasc Surg. 1996; 12(2):207-213.

26. Farooq Z, Mehmood A, Saeed S, Raja KM, Azam Khan MN, Murtaza B. Early versus late Arteriovenous fistulae: Impact on failure rate. J Ayub Med Coll Abbottabad. 2010; 22(3): 179-181.

27. Smith GE, Gohil R, Chetter IC. Factors affecting the patency of arteriovenous fistulas for dialysis access. J Vasc Surg. 2012; 55(3):849-855.

28. Vahedian J, Jalayifar AM, Keramati MR, Nabavizadeh F, Vahedian M. Primary Success of bifurcated vein patch arteriovenous fistula and Brescia-Cimino methods. Iran J Kidney Dis. 2012; 6(2):124-128.

29. Tannuri U, Aoun Tannuri AC. Experience with arteriovenous fistulas for chronic hemodialysis in children: technical details and refinements. Clinics. 2005; 60(1):37-40.

30. Leapman SB, Boyle M, Pescovitz MD, Milgrom ML, Jindal RM, Filo RS. The arteriovenous fistula for haemodialysis access: gold standard or archaic relic? Am Surg. 1996;62(8):652-657.

31. Monroy-Cuadros M, Yilmaz S, Salazar-Bañuelos A, Doig C. Independent prediction factors for primary patency loss in arteriovenous grafts within six months. J Vasc Access. 2012;13(1):29-35.

32. Lumsden AB, MacDonald MJ, Kikeri DK, Harker LA, Allen RC. Haemodialysis access graft stenosis: percutaneous transluminal angioplasty. J Surg Res. 1997; 68(2):181-185.

33. Sands, JJ. Vascular access: The past, present and future. Blood Purif .2009;27:22-27.

34. Hirth RA, Turenne MN, Woods JD, Young EW, Port FK, Pauly MV, Held PJ: Predictors of type of vascular access in haemodialysis patients. JAMA.1996; 276:1303-1307.

35. Sands J, Miranda C: Optimizing haemodialysis access: A teaching tool. Nephrol News Issues. $1996 ; 10: 16-27$.

36. Silva MB, Hobson RW, Pappas PJ, Jamil Z, Araki CT, Goldberg MC, Gwertzman G, Padberg FT: A strategy for increasing use of autogenous haemodialysis access procedures: Impact of preoperative noninvasive evaluation. J Vasc Surg.1998; 27:302-308.

Table-1. Causes of chronic kidney disease in patients who underwent A-V Fistula surgery $(n=110)$

\begin{tabular}{|l|c|c|}
\hline \multicolumn{1}{|c|}{ Causes of Chronic kidney disease } & Frequency & Percentage \\
\hline Diabetes Mellitus & 45 & $41 \%$ \\
\hline Hypertension & 32 & $29 \%$ \\
\hline Both DM \& hypertension & 16 & $14.5 \%$ \\
\hline Obstructive nephropathy & 5 & $4.5 \%$ \\
\hline Chronic interstitial nephritis & 5 & $3.6 \%$ \\
\hline Chronic glomerulonephritis & 5 & $4.5 \%$ \\
\hline Renovascular disease (ischemic nephropathy) & 1 & $0.9 \%$ \\
\hline Polycystic disease & 1 & $0.9 \%$ \\
\hline
\end{tabular}


Table- 2. Demographic and Clinical information of the patients in whom AVF adequacy could be determined as per our Fistula adequacy prospective criteria. ( $N=110)$

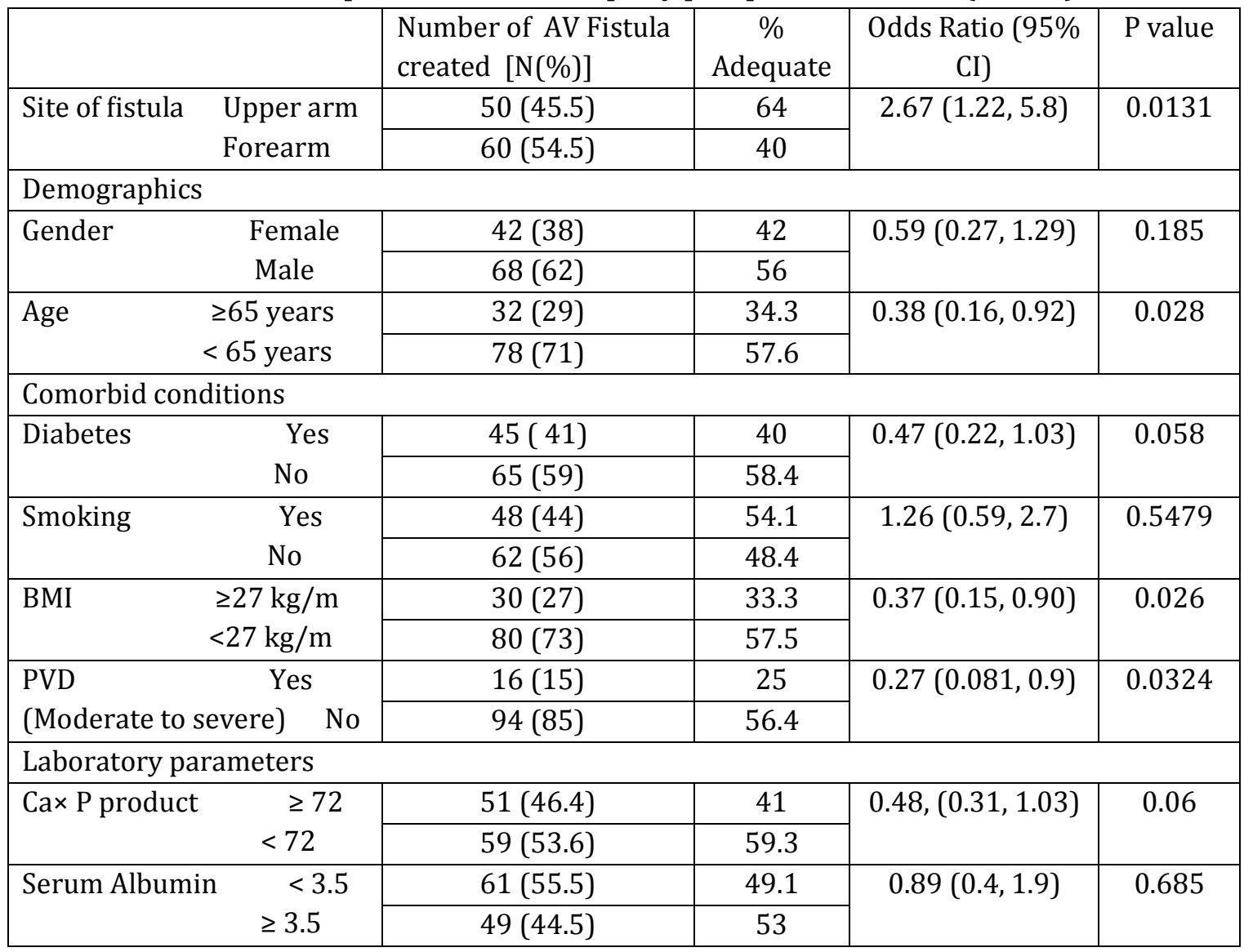

Abbreviations are: CI ( confidence interval) , AVF (arterio venous fistula), BMI (body mass index), PVD (peripheral vascular disease), Ca× P product (calcium phosphorus product).

Table -3. Distribution of Fistula Adequacy Rates according to the Anatomic Locations (Upper arm versus Forearm fistulas).

\begin{tabular}{|c|c|c|c|c|}
\hline & \multicolumn{2}{|l|}{ Upper arm } & \multicolumn{2}{|l|}{ Forearm } \\
\hline & $\begin{array}{c}\text { Number of AV Fistula } \\
\text { created }[\mathrm{N}(\%)]\end{array}$ & $\begin{array}{c}\% \\
\text { Adequate }\end{array}$ & $\begin{array}{c}\text { Number of AV Fistula } \\
\text { created }[\mathrm{N}(\%)]\end{array}$ & $\begin{array}{c}\% \\
\text { Adequate }\end{array}$ \\
\hline Females $(n=42)$ & $27(64.3)$ & 59.2 & $15(35.7)$ & 13.3 \\
\hline Male $(n=68)$ & $30(44)$ & 60 & $38(56)$ & 52.6 \\
\hline Age $\geq 65(n=32)$ & $16(50)$ & 56.3 & $16(50)$ & 12.5 \\
\hline Age $<65(n=78)$ & $30(38.5)$ & 60 & $48(61.5)$ & 56.3 \\
\hline Diabetics $(n=45)$ & $24(53.3)$ & 54.2 & $21(46.7)$ & 24 \\
\hline Nondiabetics $(n=65)$ & $35(54)$ & 65.7 & $30(46)$ & 50 \\
\hline $\mathrm{BMI} \geq 27 \mathrm{~kg} / \mathrm{m}(\mathrm{n}=30)$ & $18(60)$ & 39 & $12(40)$ & 25 \\
\hline $\mathrm{BMI}<27 \mathrm{~kg} / \mathrm{m}(\mathrm{n}=80)$ & $44(55)$ & 68 & $36(45)$ & 44.4 \\
\hline Cax $P>72(n=51)$ & $30(59)$ & 53.3 & $21(41)$ & 24 \\
\hline $\operatorname{Ca} \times \mathrm{P} \leq 72(\mathrm{n}=59)$ & $34(58)$ & 61.8 & $25(42)$ & 56 \\
\hline $\begin{array}{l}\text { PVD (Moderate to } \\
\text { severe) } \quad(n=16)\end{array}$ & $10(62.5)$ & 40 & $06(37.5)$ & 00 \\
\hline
\end{tabular}


Table- 4. Comparison of Subgroup analysis of Clinical and demographic information of the patients in whom AVF adequacy could be determined at the end of 3rd and 6th month.

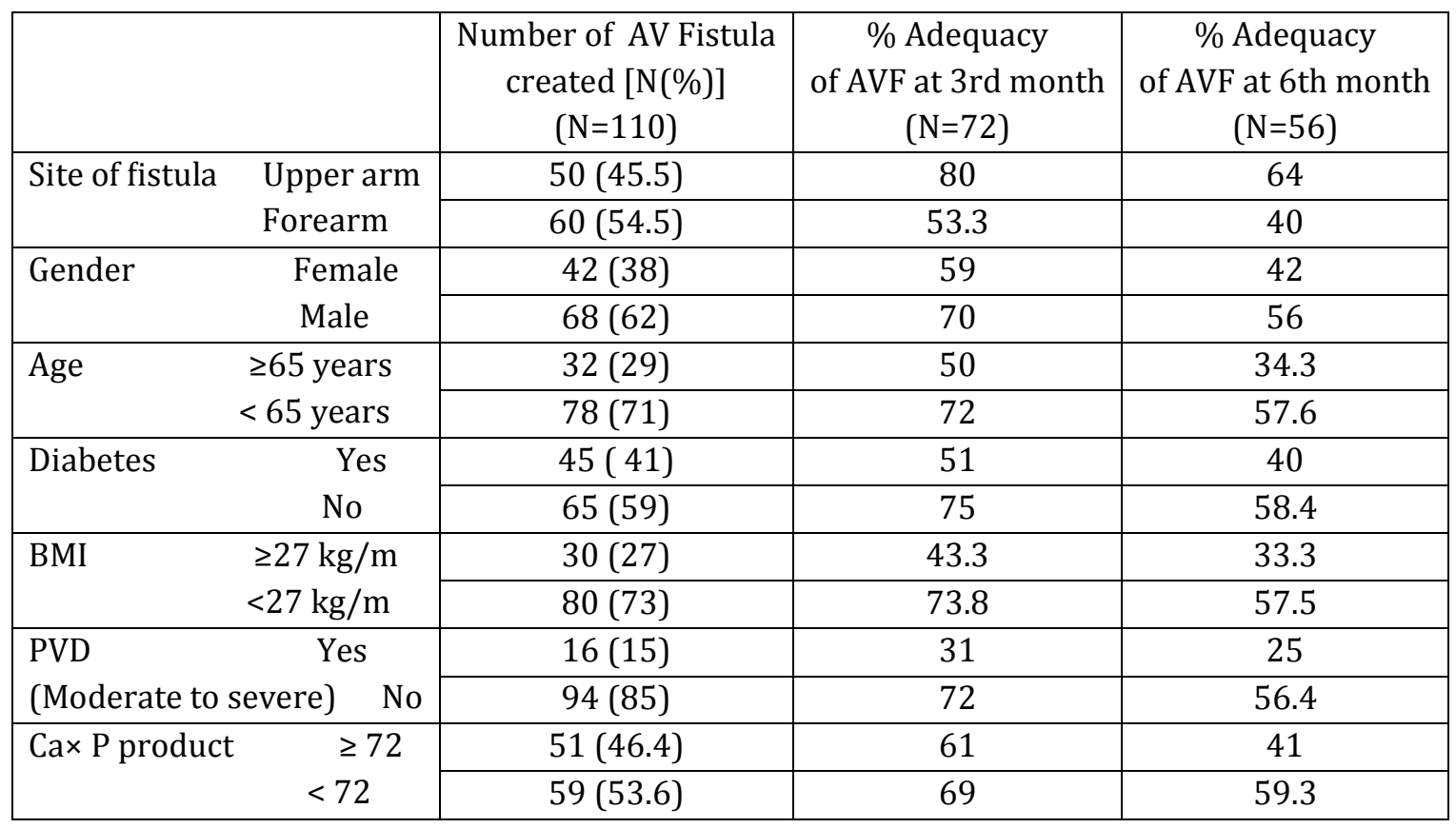

